\title{
Ictal asystole with convulsive syncope mimicking secondary generalisation: a depth electrode study
}

\author{
A O Rossetti, B A Dworetzky, J R Madsen, O Golub, J A Beckman, E B Bromfield
}

J Neurol Neurosurg Psychiatry 2005;76:885-887. doi: 10.1136/jnnp.2004.051839

Ictal bradycardia is rare and its localising value is debated. Bradyarrhythmias are, however, important because of their potential connection to sudden death and ability to affect clinical seizure manifestations. Cerebral hypoperfusion induces loss of consciousness, at times with myoclonic jerks, whose clinical differentiation from a generalised convulsive seizure may prove difficult.

Two invasive and five surface monitored seizures recorded over two years in a 51 year old woman with post-traumatic epilepsy characterised by seizure-triggered asystole were analysed. All seven seizures showed left temporal onset. Both intracranially recorded events started in the left anterior hippocampus/amygdala, spreading to the contralateral hippocampus in 35 and 25 seconds. Within 10 seconds an electrocardiogram showed asystole lasting 21 and $28 \mathrm{sec}^{-}$ onds, associated with suppression of recorded cerebral electrical activity, except a polyspike suppression pattern remaining in the hippocampi. Clinically, the patient, concomitantly with the cerebral suppression, developed myoclonic twitches of the limbs. A dual chamber cardiac pacemaker was implanted; at 11 months follow up, the patient has experienced only infrequent partial seizures, with none involving falls or shaking.

Left temporal lobe seizures produced convulsive syncope initiated by ictal asystole. These observations suggest that intertemporal spread is necessary, though not sufficient, to produce bradycardia and asystole. Furthermore, pacemakers may decrease seizure severity, as well as potentially protect against malignant bradyarrhythmias.

$\mathrm{T}$ he cerebral cortex exerts an important influence over autonomic activity. ${ }^{12}$ Seizures, as paroxysmal modifications of cortical activity, may induce dramatic changes in autonomic parameters that may be involved in the aetiology of sudden unexpected death in patients with epilepsy. ${ }^{1}$ It has been proposed, following studies with cortical electrical stimulation, ${ }^{2}$ amobarbital infusion, ${ }^{3}$ and lateralised electroconvulsive therapy, ${ }^{4}$ that activation of the right insula induces predominantly a tachycardic reaction, and of the left insula, bradycardia. With focal seizures, therefore, one would expect a roughly equivalent incidence of bradycardia and tachycardia. However, tachyarrhythmias represent a frequent finding, whereas bradycardia and its extreme form, asystole, are exceptional..$^{5-7}$ Furthermore, the localising value of bradycardia and asystole is still debated, ${ }^{1}$ since some small series or case reports ${ }^{6-11}$ postulated an association with left temporal seizures, whereas other authors did not confirm this. ${ }^{12-15}$

Pathophysiologically, cerebral hypoperfusion induces loss of consciousness, at times with myoclonic jerks (convulsive syncope). Differentiation of the latter from a generalised convulsive seizure may prove to be difficult even for experienced clinicians. ${ }^{810} 1316$

We report a patient, observed episodically over a two year period with both scalp and intracranial electrodes, with bradycardia and asystole induced by temporal lobe epileptic activity. Her electrophysiological pattern may help illuminate the complex mechanisms underlying the relation between cortical and subcortical autonomic centres.

\section{CASE REPORT}

A 51 year old woman sustained a left occipital fracture from a bicycle accident when she was 11 years old, resulting in an acute post-traumatic generalised convulsive seizure. She reported only two episodes with loss of awareness between the ages of 13 and 47 years, one during her first pregnancy. She had no previous cardiac disease, and family history was negative for neurological and cardiac disorders. At age 47 years, she began to experience paroxysmal events of confusion, preceded by dysesthesia in the left arm, nausea, muffled sounds in both ears, and lightheadedness, occasionally with loss of consciousness and convulsive movements. These episodes rapidly increased in frequency to one per week. Successive monotherapy and combination trials of phenytoin, valproate, topiramate, lamotrigine, oxcarbazepine, levetiracetam, and zonisamide allowed only incomplete control of her symptoms.

Magnetic resonance imaging (MRI) showed foci of encephalomalacia in the right hemisphere (inferior frontal gyrus, temporal pole, and anterior parietal lobe). Interictal positron emission tomography (PET) disclosed hypometabolism in the same regions, and magnetoencephalography showed interictal discharges with a right central dipole. Intracarotid amobarbital test showed exclusive left language lateralisation and better memory performance on the left side. Two long term video surface electroencephalographic (EEG) monitoring studies performed in November 2001 and February 2002 (at age 49 years) recorded five left temporal seizures. Four of them were associated with bradycardia, with maximal pauses of two to four seconds; in two, transitory mild tachycardia of $100-130$ beats per minute preceded the bradycardia.

Because of discordance in the results of this evaluation, we performed invasive monitoring with bilateral longitudinally inserted medial temporal depth electrodes and right dorsolateral frontoparietal subdural strips in October 2003, with concomitant recording from left temporal and frontopolar surface electrodes. Following antiepileptic drug (AED) taper, the patient experienced two electroclinical episodes from sleep. Both seizures started in the left anterior hippocampus/ amygdala with low voltage fast activity rapidly followed by polyspikes and rhythmic sharp activity (fig $1 \mathrm{~A}$ ), and then spread gradually to the contralateral hippocampus after 35 and 25 seconds, respectively (fig $1 \mathrm{~B}$ ). Within 10 seconds of

Abbreviations: ECG, electrocardiogram; EEG, electroencephalogram 
contralateral hippocampal spread, the electrocardiogram (ECG) showed progressive increase of the RR interval, evolving within seven seconds to asystole lasting 21 and 28 seconds (fig 1B, C); QRS complexes returned within one second of the last left hippocampal discharge (fig 1C). We did not observe any preceding tachycardia. Strip electrodes over the right frontoparietal lobe were not involved. Simultaneous scalp recordings showed rhythmic activity over the left temporal lobe starting 23 seconds after the beginning of the electrical seizure assessed by depth electrodes in both seizures (fig 1B). During the asystole, there was generalised suppression of intracerebral electrical activity, except for rhythmic theta and delta and then burst suppression in the right hippocampus, and a polyspike suppression pattern in the left hippocampus (fig $\mathrm{IC}$ ). Clinically, the patient was motionless until 20 and eight seconds after the beginning of asystole, and then showed, in parallel with the suppression pattern, myoclonic twitches of the limbs lasting for more than 10 seconds (fig $\mathrm{lC}$ ). Both postictal phases were characterised by recovery over more than 15 minutes.

Cardiological evaluation, including 12-lead interictal ECG and echocardiography, was normal. We implanted a dual chamber pacemaker in the patient but resective brain surgery was not done. Eleven months later, the patient remains on valproate and zonisamide, and has reported only infrequent, isolated episodes of somatosensory phenomena but no generalised jerking or falls. She also feels mentally clearer and better able to function. The pacemaker has been interrogated on two occasions since implantation. During the most recent visit, we noted several bradycardic episodes and pacing was required $8 \%$ of the time.

\section{COMMENT}

Invasive EEG monitoring confirmed the diagnosis of left temporal lobe epilepsy, and suggested that the reported "generalised convulsions" most likely were episodes of convulsive syncope following cerebral hypoperfusion. It is notable that asystole developed in both episodes from a rapidly progressive bradycardia shortly after ictal discharges had spread from the left to the right hippocampus. This may suggest that a particular propagation pattern in our patient led to bilateral activation of mesial temporal structures related to the insula and subcortical nuclei critical to autonomic control, particularly the hypothalamus. ${ }^{1}$ Supporting this view, two previous reports of intracranial recording during ictal asystole briefly mentioned that the latter began after the seizure had spread from the left to the right temporal lobe, although the exact timing was not specified..$^{12} 17$ Seizure spread from one hippocampus commonly involves propagation to the ipsilateral neocortex followed by contralateral hippocampal activation, probably after homologous neocortical involvement through the anterior commissure or the corpus callosum. ${ }^{18}$ However, direct propagation between hippocampi, without neocortical recruitment, has been demonstrated, leading to the assumption of the existence of a hippocampal commissure, ${ }^{18}$ a controversial hypothesis, ${ }_{19}^{19}$ which, however, has an anatomical correlate. ${ }^{20}$ Unfortunately, we were not able to record with depth electrodes from the neocortical portions of the temporal lobes. Nevertheless, left temporal surface EEG showed rhythmic activity before involvement of the right hippocampus, a finding that may suggest lateral temporal lobe recruitment at least on the left side, and thus seizure spread through the anterior commissure or the corpus callosum. Of note, the right frontal lobe was not involved. Indeed, stimulation studies of the frontal lobe in humans failed to show a consistent modification of the heart rate..$^{21}$ Our recordings limited to scalp EEG showed how variable the occurrence of ictal tachycardia and bradycardia can be, even

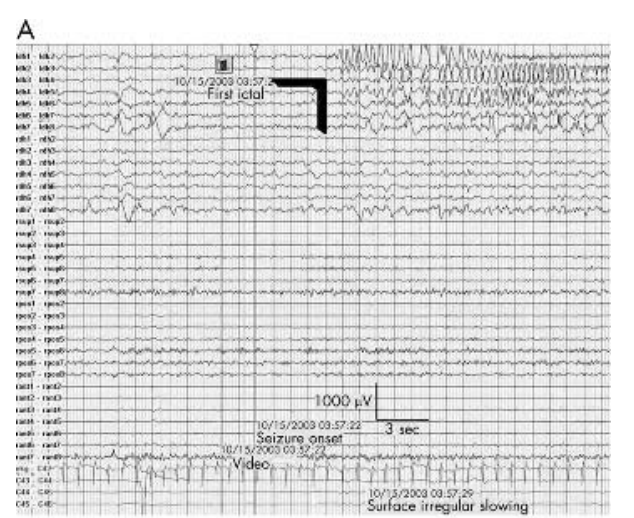

B
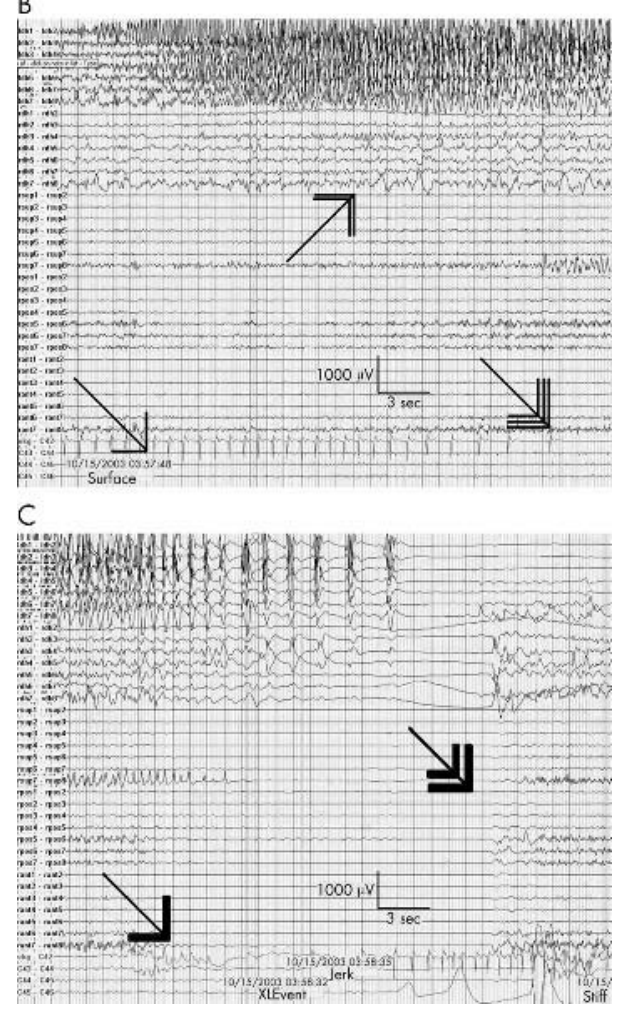

Figure 1 Consecutive recording of the ictal asystole during the first intracranially recorded seizure. Electrodes were labelled as follows: Idh, left depth hippocampal electrode; rdh, right depth hippocampal electrode; rsup, right superior parietofrontal subdural strip; rpos, right poster inferior parietofrontal subdural strip; rant, right anterior frontal subdural strip; $\mathrm{C} 43=\mathrm{FPl} ; \mathrm{C} 44=\mathrm{F} 7 ; \mathrm{C} 45=\mathrm{T} 3 ; \mathrm{C} 46=\mathrm{T} 5$. Calibration for intracranial recording is given on the images. (A) Beginning of the ictal discharge in the most anterior leads of left depth electrode, corresponding to anterior hippocampus/amygdala (arrowhead) spreading rapidly to the whole hippocampus. (B) Rhythmic activity appearing on left hemispheric surface leads (simple arrow) preceding gradual spread to the right hippocampus (double arrow). Bradycardia, rapidly evolving into asystole (triple arrow), lasting for 21 seconds. Channel rsup7-rsup8 shows an artefact. (C) Flattening of electrical activity, apart from a burst suppression pattern persisting in the hippocampi, especially on the left. First body and limb jerk towards the end of the asystole (thick arrow). After the return of cardiac activity, recovery of the cerebral electrical activity but with persistent suppression in the left anterior hippocampal leads. Muscle artefact (double thick arrow) on the ECG and superficial EEG channels corresponding to stiffening.

in clinically similar seizures occurring in the same patient. This is not a surprising finding, as the timing of seizure spread has been found to vary considerably between events in the same subjects. ${ }^{18}$ This variability may help to explain why 
bradycardia and asystole are encountered in only a small subgroup of seizures. The occurrence of asystole during the invasive monitoring, but only of bradycardia two years earlier during surface EEG monitoring, may be due to progression of seizure intensity, or to a "stochastic" distribution of such episodes. An alternative to the hypothesis that cardiac effects were mediated through interhippocampal seizure spread, perhaps influenced by neocortical lesions in our patient, is synchronous unilateral spread from the left temporal lobe to subcortical vegetative structures and to the right hippocampus; however, this would not explain the rarity of this phenomenon, or why in the literature several cases of bradycardia-asystole are reported to arise from the right hemisphere. Furthermore, the patient was not taking any medication, particularly carbamazepine or phenytoin, known for inducing arrhythmia, and she did not have a history of heart disease. ${ }^{10}$

An additional feature of potential importance is our patient's long postictal recovery phase despite a relatively limited epileptic discharge. One could hypothesise that this resulted from a direct neuronal insult caused by simultaneous ictal hypermetabolism coupled with decreased substrate supply. Our patient's perception of improved mental functioning after pacemaker implantation supports this view, although there was also a decrease in medications.

This case report underlines the importance of concomitant intracranial EEG/ECG registration. The timing of electrical changes (that is, electrographic seizure preceding ECG abnormality) recorded through intracranial electrodes suggests that previous reports addressing this issue and describing ECG abnormalities preceding seizure onset ${ }^{56}$ could be biased by the surface EEG recording. Finally, pacemaker implantation may prove not only to be potentially lifesaving in this setting, ${ }^{9}{ }^{13} 16$ as is highlighted by the device interrogation in our patient, but also can reduce clinical seizure severity as well as possible neurological morbidity prior to resective brain surgery.

\section{Authors' affiliations}

A O Rossetti, B A Dworetzky, O Golub, E B Bromfield, Epilepsy Monitoring Unit, Department of Neurology, Brigham and Women's Hospital, Harvard Medical School, Boston, MA, USA

J R Madsen, Department of Neurosurgery, Brigham and Women's Hospital, Harvard Medical School, Boston, MA, USA

J A Beckman, Division of Cardiovascular Medicine, Brigham and Women's Hospital, Harvard Medical School, Boston, MA, USA

Dr Rossetti is supported by the Swiss National Science Foundation and the SICPA Foundation, Prilly, Switzerland.

Competing interests: none declared
Correspondence to: E B Bromfield, Brigham and Women's Hospital, Department of Neurology, Division of Epilepsy and EEG, 75 Francis Street, Boston, MA 02115, USA; ebromfield@partners.org

Received 11 August 2004

Revised version received 9 September 2004

Accepted 15 September 2004

\section{REFERENCES}

1 Devinsky O. Effects of seizures on autonomic and cardiovascular function. Epilepsy Curr 2004;4:43-6.

2 Oppenheimer SM, Gelb A, Girvin JP, et al. Cardiovascular effects of human insular cortex stimulation. Neurology 1992;42:1727-32.

3 Zamrini EY, Meador KJ, Loring DW, et al. Unilateral cerebral inactivation produces differential left/right rate heart responses. Neurology 1990:40:1408-11.

4 Swartz CM, Abrams R, Lane RD, et al. Heart rate differences between right and left unilateral electroconvulsive therapy. J Neurol Neurosurg Psychiatry 1994;57:97-9.

5 Blumhardt LD, Smith PE, Owen L. Electrocardiographic accompaniments of temporal lobe epileptic seizures. Lancet 1986;1:1051-6.

6 Leutmezer F, Schernthaner C, Lurger S, et al. Electrocardiographic changes at the onset of epileptic seizures. Epilepsia 2003;44:348-54.

7 Nei M, Ho RT, Sperling MR. EKG abnormalities during partial seizures in refractory epilepsy. Epilepsia 2000;41:542-8.

8 Kowalik A, Baver J, Elger CE. Asystolische Anfälle. Nervenarzt 1998;69:151-7.

9 Locatelli ER, Varghese JP, Shuaib A, et al. Cardiac asystole and bradycardia as a manifestation of left temporal lobe complex partial seizure. Ann Intern Med 1999;130:581-3.

10 Rocamora R, Kurthen M, Lickfett L, et al. Cardiac asystole in epilepsy: clinical and neurophysiologic features. Epilepsia 2003;44:179-85.

11 Lim EC, Lim SH, Wilder-Smith E. Brain seizes, heart ceases: a case of ictal asystole. J Neurol Neurosurg Psychiatry 2000;69:557-9.

12 Ghearing GR, Britton JW. The ictal bradycardia syndrome: clinical features, localization and results of cardiac pacing [abstract]. Neurology 2004;62(suppl 5):A250.

13 Liedholm LJ, Gudjonsson O. Cardiac arrest due to partial epileptic seizures. Neurology 1992;42:824-9.

14 Reeves AL, Nollet KE, Klass DW, et al. The ictal bradycardia syndrome. Epilepsia 1996;37:983-7.

15 Wilder-Smith E, Lim SH. Heart rate changes during partial seizures: a study among Singaporean patients. BMC Neurology 2001;1:5

16 Linzer M, Grubb BP, Ho S, et al. Cardiovascular causes of loss of consciousness in patients with presumed epilepsy. A cause of the increased sudden death rate in people with epilepsy? Am J Med 1994;96:146-54.

17 Devinsky O, Pacia S, Tatambothla G. Bradycardia and asystole induced by partial seizures: a case report and literature review. Neurology 1997;48:1712-14.

18 Spencer SS, Williamson PD, Spencer DD, et al. Human hippocampal seizure spread studied by depth and subdural recording: the hippocampal commissure. Epilepsia 1987;28:479-89.

19 Lieb JP, Babb TL, Engel J Jr, et al. Propagation pathways of interhemispheric seizure discharges compared in human and animal hippocampal epilepsy. In: $J$ Engel Jr, ed. Fundamental mechanisms of human brain. New York: Raven Press, 1987:165-70.

20 Gloor P, Salanova V, Olivier A, et al. The human dorsal hippocampal commissure. An anatomically identifiable and functional pathway. Brain 1993;1 16:1249-73.

21 Pool JL, Ransohoff J. Autonomic effects on stimulating rostral portions of cingulate gyri in man. J Neurophysiol 1949;12:385-92.

22 Delgado JMR. Circulatory effects of cortical stimulation. Physiol Rev 1960;40:146-70. 